\title{
Study on Growth and Change of Solid Particles with Water Flow in Oilfield Water-Injection Pipeline
}

\author{
Guiliang LI, Changjun LI, Jie WANG, Nanning XIA, Xiuling CHEN
}

\begin{abstract}
The solid particles in oilfield water-injection pipelines with water flow will continuously grow and change, and the oversized solid particles may block the pores of the formation and reduce the oilfield recovery efficiency. Therefore, the study on the growth and change to solid particles during transportation has become a question of interest in oilfields. However, there is little research on this question currently. Therefore, on the basis of the liquid-solid two-phase flow model and the particle population balance model, a growth and change model of solid particles in long-distance water-injection pipelines flowing along water was established in this paper in consideration of the injected water temperature drop along the path, as well as the growth, coalescence, breakage and deposition of particles. Comparison of the field test results indicated that the average error of the particle size distribution fitting degree calculated by the model is $6.9 \%$, and the average error of median diameter is $4.1 \%$. This model was used for analyzing the impact of the flow rate, temperature and median diameter of the united station outlet in a block oilfield of Shengli Oilfield on the solid particle size of the wellheads, and the critical flow rate, temperature and median diameter of the united station outlet were predicted when the median diameter at the wellheads meets the injection requirement $(<2 \mu \mathrm{m})$. The establishment of this model cannot only be used for the study on the growth and change to solid particles in water-injection pipelines flowing along water, but also provides a technical reference for the study on the growth and change to low-concentration solid particles accompanying flow in long-distance liquid/gas phase pipelines.
\end{abstract}

Keywords: injected water; long-distance pipeline; liquid-solid two-phase flow model; particle population balance model; particle size distribution

\section{INTRODUCTION}

Produced water in oilfields will be injected into the formation after being processed by the united station to maintain formation pressure and reduce the impact on local environments [1]. During the transportation process, the solid particles in the injected water will continuously grow and change due to chemical reaction growth or coalescence of particles [2]. A large number of large-size particles, if generated, may block the pores of the formation and reduce the oilfield recovery efficiency [3]. Therefore, the study on the growth and change to solid particles during transportation of injected water has become a question of interest in oilfields. This problem is also manifested in the pipelining of flour, sugar and mud, etc. [4, 5].

To describe the growth and change to solid particles in the liquid phase pipeline, the researchers used the method of coupled liquid-solid two-phase flow model and particle population balance model $[5,6]$. Researches on the theory of liquid-solid two-phase flow began in the 1940s [7]. Single-particle dynamic model first appeared. This model holds that solid particles have no effect on flow pattern and have independent, stable and regular movement in flow field. In view of interphase interaction that is not considered in single-particle dynamic model, particle trajectory model is proposed by Crowe [8] based on the research result of gas-droplet two-phase flow. Relative to single-particle dynamic model, this model adds source item of liquid-solid interaction to hydrodynamic equation so that it is closer to flow reality of two-phase flow. The above two models treat fluid and particle as continuous media and discrete media respectively. Soo [9] proposed a dispersion model, assuming that the discrete particles are treated as continuous media (pseudo-fluid). This model uses the continuous medium theory to study the movement of granular media and the turbulent diffusion between particle phase and fluid is considered. In addition, Spalding [10] also proposed a single fluid model, holding that there is no relative motion between solid particles and fluid, and the particle phase should satisfy the continuity equation only. As for the issue that the single fluid model is too simplified, Bowen [11] proposed a two-fluid model. In this model, the turbulent diffusion of each phase, interaction between solid particles and interaction between solid particles and fluid are considered. This model also holds that the kinetic and thermodynamic parameters of particle phase are considered as spatial continuous distribution function. In oil industry, the two-fluid model is generally used in the study of sulfur deposition [12], wax deposition [13] and hydrate transportation [5], but the solid particles in water reinjection pipeline are less researched.

The liquid-solid two-phase flow model mainly depicted the impact of the interaction between the liquidphase solvent and the solid-phase solute on the flow field during the flow process, and failed to consider the changes to the development of solid particle. In order to describe the growth and change to solid particles and improve liquid-solid two-phase flow model, Hulburt \& Katz [14] proposed the particle population balance model (PBM) according to the law of conservation of particle mass. The model can describe the primary crystallization (nucleation \& growth), secondary crystallization (coalescence \& breakage) and deposition of solid particles [5]. Accurate modeling of these dynamic events constitutes the key to application of the model. Arrhenius put forward the crystallization rate equation in accordance with the concentration and energy changes of the reaction system [15]. By correlating the particle growth rate with the particle size, Abegg et al. [16] proposed the ASL growth model. On the grounds of different solid particle coalescence mechanisms, Smoluchowski, Camp, Abrahamson respectively proposed the particle coalescence model when the particles were mainly controlled by Brownian action, laminar flow and turbulence $[5,17]$. After comprehensive consideration of the mechanism of particle wear breakage and fragmentation breakage, Fan [18] proposed a particle breakage model based on the moment method. By considering the impact of diffusion and gravity action on 
the deposition of solid particles in the flow field, Park [19] proposed a corresponding solid particle deposition model. Despite the extensive application studies on the particle population balance model, these studies usually only considered the change to solid particles with time and flow rate and ignored the impact of Brownian diffusion on small solid particles $(<1 \mu \mathrm{m})[5,6]$. This will be unable to fully reflect the growth and change to small particles affected by Brownian motion in the injected water.

As a result, as to the growth and change to solid particles in the water-injection pipeline, by combining the injected water operating conditions and test results of particle size and content of solid particles in a certain block of Shengli Oilfield, based on the liquid-solid two-phase flow model and the particle population balance model, we establish a growth and change model for solid particles in long-distance water-injection pipelines. In the subsequent second section, a growth and change model for solid particles in long-distance water-injection pipeline is established. The third section is the solving algorithm for the model. The fourth section is the application result and discussion of the new model. The last section is the summary and conclusion of the results of this study.

\section{MATHEMATICAL MODEL}

\subsection{Liquid-Solid Two-Phase Flow Control Equation}

The mass conservation equation, the momentum conservation equation and the energy conservation equation should be satisfied in the liquid-solid two-phase flow process, as shown in Eqs. (1) to (6), respectively.

For liquid and solid phase continuity Eq. (5), see Eqs. (1) and (2) respectively.

$$
\begin{gathered}
\frac{\partial \alpha_{1} \rho_{1}}{\partial t}+\nabla\left(\alpha_{1} \rho_{1} \boldsymbol{V}_{1}\right)=\dot{m}_{1} \\
\frac{\partial \alpha_{\mathrm{s}} \rho_{\mathrm{s}}}{\partial t}+\nabla\left(\alpha_{\mathrm{s}} \rho_{\mathrm{s}} \boldsymbol{V}_{\mathrm{s}}\right)=\dot{m}_{\mathrm{s}}
\end{gathered}
$$

where $\alpha_{111}$ and $\alpha_{\mathrm{s}}$ are the volume fractions of the liquid phase and the solid phase, respectively, with their sum of 1. $\rho_{\mathrm{l}}$ and $\rho_{\mathrm{s}}$ represent the density of the liquid phase and the solid phase, respectively, $\mathrm{kg} / \mathrm{m}^{3} . \boldsymbol{V}_{1}$ and $\boldsymbol{V}_{\mathrm{s}}$ represent velocity vector of the liquid phase and the solid phase respectively, m/s. $\dot{m}_{1}$ and $\dot{m}_{\mathrm{s}}$ represent the mass source term of the liquid phase and the solid phase, respectively, with their sum of 0 .

For the liquid and solid phase momentum conservation equations (6), see Eqs. (3) and (4) respectively.

$$
\begin{aligned}
& \frac{\partial\left(\alpha_{1} \rho_{\mathrm{l}} \boldsymbol{V}_{1}\right)}{\partial t}+\alpha_{1} \rho_{\mathrm{l}} \boldsymbol{V}_{1}\left(\nabla \cdot \boldsymbol{V}_{1}\right)+\left(\boldsymbol{V}_{1} \cdot \nabla\right) \alpha_{1} \rho_{\mathrm{l}} \boldsymbol{V}_{1}= \\
& =\nabla \cdot \boldsymbol{\tau}_{1}+\alpha_{1} \rho_{\mathrm{l}} \boldsymbol{g}+f_{i}+\dot{\boldsymbol{M}}_{1} \\
& \frac{\partial\left(\alpha_{\mathrm{s}} \rho_{\mathrm{s}} \boldsymbol{V}_{\mathrm{s}}\right)}{\partial t}+\alpha_{\mathrm{s}} \rho_{\mathrm{s}} \boldsymbol{V}_{\mathrm{s}}\left(\nabla \cdot \boldsymbol{V}_{\mathrm{s}}\right)+\left(\boldsymbol{V}_{\mathrm{s}} \cdot \nabla\right) \alpha_{\mathrm{s}} \rho_{\mathrm{s}} \boldsymbol{V}_{\mathrm{s}}= \\
& =\nabla \cdot \boldsymbol{\tau}_{\mathrm{s}}+\alpha_{s} \rho_{\mathrm{s}} \boldsymbol{g}-f_{i}+\dot{\boldsymbol{M}}_{\mathrm{s}}
\end{aligned}
$$

where $\tau_{1}$ and $\boldsymbol{\tau}_{\mathrm{s}}$ are the surface force tensors of the liquid phase and solid phase control volumes, respectively, $\mathrm{N} / \mathrm{m}^{2}$. $\boldsymbol{g}$ is the mass force vector, N. $f_{i}$ is the interaction force (e.g. drag force, lift, virtual mass force and Basset force) between the solid particles and the liquid phase [20]. $\dot{\boldsymbol{M}}_{1}$ and $\dot{\boldsymbol{M}}_{\mathrm{s}}$ are liquid phase and solid phase momentum vectors caused by mass transfer respectively, $\mathrm{N} / \mathrm{m}^{3}$.

For the liquid and solid phase energy conservation Eq. (5), see Eqs. (5) and (6), respectively.

$$
\begin{aligned}
& \frac{\partial \alpha_{1} \rho_{1} e_{1}}{\partial t}+\left(\nabla \cdot \alpha_{1} \rho_{1} e_{1} V_{1}\right)=\nabla \cdot\left(\alpha_{1} \rho_{1} k_{1} \nabla T_{1}\right)+\Psi_{1}+q_{1} \\
& \frac{\partial \alpha_{\mathrm{s}} \rho_{\mathrm{s}} e_{\mathrm{s}}}{\partial t}+\left(\nabla \cdot \alpha_{\mathrm{s}} \rho_{\mathrm{s}} e_{\mathrm{s}} \boldsymbol{V}_{\mathrm{s}}\right)=\nabla \cdot\left(\alpha_{\mathrm{s}} \rho_{\mathrm{s}} k_{\mathrm{s}} \nabla T_{\mathrm{s}}\right)+\Psi_{\mathrm{s}}+q_{\mathrm{s}}
\end{aligned}
$$

where $e_{1}$ and $e_{\mathrm{s}}$ are the energy of the liquid phase and solid phase control volumes, respectively, including internal energy and kinetic energy. $\Psi=2 \mu \boldsymbol{S}: \boldsymbol{S}$, where $\boldsymbol{S}$ is the strain rate tensor. $q_{1}$ and $q_{\mathrm{s}}$ are the energy transfer source term of liquid phase and solid phase respectively.

For long-distance reinjection water pipes, the application Eqs. (1) to (6) need further discussion, see section 2.3 for details.

\subsection{Particle Population Balance Model}

Population balance model proposed by Smoluchowski [17] is as shown in Eq. (7).

$$
\frac{\partial n(v, x, t)}{\partial t}+\nabla(V n(v, x, t))-\nabla \cdot\left(D_{x} \nabla n(v, x, t)\right)=S(v, x, t)(7)
$$

where, $n(v, x, t)$ is the particle number density function, $\mathrm{a} / \mathrm{m}^{3} . V$ is the average rate of the $v$ particle in the $i$ direction, $\mathrm{m} / \mathrm{s} . D_{x}$ is the diffusion coefficient. $S(v)$ is the kernel function of dynamic events of particles, such as growth, coalescence, breakage and deposition kernel functions.

(1) Diffusion coefficient. Because of the different Knudsen number $(K n)$ [21] ranges, there are different computing models for the diffusion coefficient $D_{x}$. Kn can be calculated by Eq. (8).

$$
K n=\frac{\lambda}{r}
$$

where $\lambda$ is the mean free path of the solvent molecule, $\mathrm{m} ; r$ is the critical nucleation radius of solid particle, $\mathrm{m}$.

When $K n<1$, Einstein [21] considers that the diffusion coefficient can be calculated according to Eq. (9).

$$
D_{x}=\frac{k_{\mathrm{B}} T C_{C}}{3 \pi \mu d_{\mathrm{p}}}
$$

where $k_{\mathrm{B}}$ is the Boltzmann constant, $1.380649 \times 10^{-23} \mathrm{~J} / \mathrm{K} . T$ is the temperature, $\mathrm{K} ; \mu$ is the liquid dynamic viscosity, $\mathrm{Pa} \cdot \mathrm{s} ; d_{\mathrm{p}}$ is the particle diameter, $\mathrm{m} ; C_{C}$ is the correction coefficient, for which Pratsinis [22] gives the expression, see Eq. (10). 


$$
C_{C}=1+K n[1.142+0.558 \exp (-0.999 / K n)]
$$

When $K n>50$, the diffusion coefficient can be calculated by Eq. (11) [21].

$$
D_{x}=\frac{k_{\mathrm{B}} T}{\frac{2}{3} d_{\mathrm{p}}^{2} \rho c \frac{\pi}{2}\left(1+\frac{\pi \alpha}{8}\right)}
$$

where $\rho$ is the liquid phase density, $\mathrm{kg} / \mathrm{m}^{3} ; c$ is the liquid phase velocity, $\mathrm{m} / \mathrm{s} . \alpha$ is the adjustment factor, which can be 0.9 .

When $1 \leq K n \leq 50$, the particle diffusion coefficient can be obtained by harmonic average of Eqs. (9) and (11) [23].

(2) Particle growth model. The particle growth model can be divided into a size-independent growth model, a size-dependent model, or a growth dispersion model. The size-dependent model considers that the growth rate of the particles is related to their particle size. The ASL sizedependent model proposed by Abegg [16] is a commonly used particle growth model, as shown in Eq. (12).

$$
G(L)=G_{0}(1+a L)^{b}
$$

where $G_{0}$ is the growth rate of the crystal nucleus, $\mathrm{m} / \mathrm{s} . L$ is the particle size, m. $a$ and $b$ are model parameters and can be obtained experimentally.

(3) Coalescence model. For particle coalescence, Smoluchowski [17] gives the coalescence mathematical expression of multi-scale particles in discrete form, and its calculus form can be obtained, see Eq. (13).

$$
\begin{aligned}
S_{\mathrm{ag}} & =\frac{1}{2} \int \beta_{\mathrm{ag}}(v, v-u) n(v, x, t) n(v-u, x, t) \mathrm{d} v- \\
& -\int \beta_{\mathrm{ag}}(v, u) n(v, x, t) n(v-u, x, t) \mathrm{d} v
\end{aligned}
$$

where, $S_{\mathrm{ag}}$ represents a coalescence source term. $\beta_{\mathrm{ag}}$ is a coalescence coefficient. $u$ represents a particle with a volume $u$. The first term on the right side of the equation represents the generation rate of coalescence and generation of the $v$ particle, and the second term represents the decay rate of the $v$ particle after coalescence with other particles.

Depending on different mechanisms of the coalescence action, the coalescence coefficient has various forms, such as Brownian coalescence and turbulence coalescence.

For Brownian coalescence, Park [23] divides it into three regions according to different $K n$.

When $K n<1$, the coalescence coefficient can be calculated by the Eq. (14).

$$
\beta_{\mathrm{ag}}(v, u)=K\left(v^{1 / 3}+u^{1 / 3}\right)\left(v^{-1 / 3}+u^{-1 / 3}\right)
$$

When $1 \leq K n \leq 50$, the coalescence coefficient model is shown in Eq. (15).

$$
\beta_{\mathrm{ag}}(v, u)=C_{\mathrm{ag}} K\left(v^{1 / 3}+u^{1 / 3}\right)\left(v^{-1 / 3}+u^{-1 / 3}\right)
$$

where $C_{\mathrm{ag}}$ is a solid particle coalescence correction term, see Eq. (16).

$C_{a g}=1+1.591 K n$

When $K n>50$, the coalescence coefficient is calculated using Eq. (17).

$\beta_{\mathrm{ag}}(v, u)=\left(\frac{3}{4 \pi}\right)^{1 / 6}\left(\frac{6 k_{\mathrm{B}} T}{\rho_{\mathrm{P}}}\right)\left(v^{1 / 3}+u^{1 / 3}\right)^{2}\left(v^{-1}+u^{-1}\right)^{2}$

where, $\rho_{\mathrm{p}}$ is the solid-phase particle density, $\mathrm{kg} / \mathrm{m}^{3}$.

For the turbulence coalescence coefficient, calculation can be performed using Eq. (18) [24].

$\beta_{\mathrm{T}}(v, u)=K\left(v^{1 / 3}+u^{1 / 3}\right)\left|v^{2 / 3}-u^{2 / 3}\right|$

The collision coefficient $K$ is calculated by equation (19) [25].

$K=\frac{2 k_{\mathrm{B}} T}{3 \mu}$

Solid particles are simultaneously subjected to Brownian action and turbulence because there are solid particles with both small size $(<1 \mu \mathrm{m})$ and large size $(\geq 1$ $\mu \mathrm{m})$ in the injected water. In this paper, Eq. (20) expresses the coalescence coefficient of the solid particles in the injected water.

$\beta_{\mathrm{Z}}(v, u)=C_{1} \beta_{\mathrm{B}}(v, u)+C_{2} \beta_{\mathrm{T}}(v, u)$

where, $\beta_{\mathrm{Z}}$ represents the coalescence coefficient of solid particles in the injected water. $C_{1}$ and $C_{2}$ respectively represent the coalescence weights of solid particles with particle size $<1 \mu \mathrm{m}$ and particle size $\geq 1 \mu \mathrm{m}$, and can be determined experimentally.

(4) Breakage model. The breakage involves breakage of basic particles and breakage of large particles of coalescence. The former is the reverse process of solid particle growth, and the latter is the reverse process of coalescence. These processes can also be referred to as attrition breakage and fragmentation breakage, respectively. The mechanism of particle breakage is not the same, but can still be written in a uniform form [18], see Eq. (21).

$$
\begin{aligned}
S_{\mathrm{br}}= & \iint a_{\mathrm{br}}(u) b_{\mathrm{br}}(u) n(u, x, t) \mathrm{d} v \mathrm{~d} u- \\
& -\int a_{\mathrm{br}}(v) n(u, x, t) \mathrm{d} v
\end{aligned}
$$

where, $S_{\mathrm{br}}$ represents the breakage function; $a_{\mathrm{br}}(u)$ represents the generation probability of large particle of breaking into $u$ particle; $b_{\mathrm{br}}(v \mid u)$ represents the small particle distribution function after breakage. The rest of symbols have the same meanings as above. 
When $S_{\mathrm{br}}$ is calculated using the moment method, $a_{\mathrm{br}}$ and $b_{\text {br }}$ can be calculated by Eqs. (22) and (23), respectively [26].

$$
\begin{aligned}
& a_{\mathrm{br}}\left(L_{i}\right)=\left\{0 \quad L=10.1 \mathrm{e}^{0.01 L_{i}^{3}} \quad L>1\right. \\
& b_{\mathrm{br}}^{k}=1+\left(L_{i}^{3}-1\right)^{k / 3}
\end{aligned}
$$

where, $L_{i}$ is the characteristic length of $i$ particle; $k$ is the order of the moment.

(5) Deposition model. Park [19] proposed a deposition kernel function that considers particle diffusion and gravity action, see Eq. (24).

$$
E(v)=E_{\mathrm{B}}(v)+E_{\mathrm{g}}(v)
$$

where, $E_{\mathrm{B}}(v)$ and $E_{\mathrm{g}}(v)$ are the deposition kernel functions controlled by particle diffusion and gravity action, respectively, see Eqs. (25) and (26).

$$
\begin{aligned}
& E_{\mathrm{B}}(v)=A v^{\frac{-2(n-1)}{3 n}} \\
& E_{\mathrm{g}}(v)=B v^{2 / 3}
\end{aligned}
$$

In Eq. (25) and Eq. (26), for $A$ and $B$ coefficients, refer to the literature [19]. $n$ is the adaptation coefficient, and can be obtained experimentally.

When particles have growth, agglomeration, fragmentation, and deposition kinetics events, the moment $S(v, x, t)$ of in Eq. (7) can be written as Eq. (27).

$$
\begin{aligned}
& \int S(v, x, t) v^{k} \mathrm{~d} v=k \sum_{i=1}^{N} w_{i} V_{i}^{k-1} G\left(V_{i}\right)+ \\
& +\frac{1}{2} \sum_{i=1}^{N} \sum_{j=1}^{N} \beta_{i j} w_{i} w_{j}\left[\left(V_{i}+V_{j}\right)^{k}-V_{i}^{k}-V_{j}^{k}\right]+ \\
& +\sum_{j=1}^{N} w_{i} a_{\mathrm{br}}\left(b_{\mathrm{br}}-V_{i}^{k}\right)-\left(A V_{i}^{k-2(n-1) / 3 n}+B V_{i}^{k+2 / 3}\right)
\end{aligned}
$$

where, $G\left(V_{i}\right)=G_{0}\left(3 V_{i} /(4 \pi)\right)^{-3}, G_{0}=1.0 \mathrm{~s}^{-1}$ can be taken [31].

\subsection{Growth and Change Model of Solid Particles in Water- Injection Pipeline}

In order to avoid safety hazards due to pressure fluctuations caused by frequent adjustment of operating conditions of injected water, the operating conditions of injected water should be stable. Therefore, the transportation process of injected water can be approximated as a steady flow. The content of solid particles in the injected water is much less than $1 \%$ of the mass of water, so the impact of solid particles on the liquid phase flow can be neglected [27]. Therefore, the injected water flow can be approximated as a single-phase liquid flow, and the continuity equation, the momentum conservation equation, and the energy conservation equation can be reduced to Eqs. (28) to (30), respectively.

$\frac{\mathrm{d}}{\mathrm{d} x}\left(\rho_{1} v_{1} A_{1}\right)=0$

$\frac{\mathrm{d}}{\mathrm{d} x}\left(\rho_{l} v_{l}^{2} A_{l}\right)=0$

$\frac{\mathrm{d}}{\mathrm{d} x}\left(\rho_{l} v_{l}^{2} A_{l}\left(H_{l}+\frac{v_{l}^{2}}{2}\right)\right)=Q_{x}$

El-Dessouky [28] proposed a semi-empirical model for calculating brine density, see Eq. (31).

$\rho=\left(A_{1} F_{1}+A_{2} F_{2}+A_{3} F_{3}+A_{4} F_{4}\right)$

Tab. 1.

The relevant parameters in the equation are shown in

If the effect of density can be neglected during the exportation of injected water, the flow behavior of the injected water can be approximated as an incompressible and stable flow.

Thus, Eq. (30) can be written as Eq. (32).

$\left(\frac{\partial h}{\partial T}\right)_{P} \frac{\mathrm{d} T}{\mathrm{~d} x}+\left(\frac{\partial h}{\partial P}\right)_{T} \frac{\mathrm{d} P}{\mathrm{~d} x}=-\frac{\mathrm{d} Q_{x}}{\mathrm{~d} x}$

After further derivation, the formula for temperature drop along the path can be obtained, see Eq. (33).

$T=T_{0}+\left(T_{Q}-T_{0}\right) e^{-a x}+D_{\mathrm{pl}} \mathrm{e}^{-a x} \int_{0}^{x} \frac{\mathrm{d} p}{\mathrm{~d} x} \mathrm{e}^{-a x} \mathrm{~d} x$

where, $T_{0}$ and $T_{Q}$ is the ambient temperature and the starting temperature, respectively, K. $D_{\mathrm{pl}}$ is the inner diameter of the pipeline, m. $a=K \pi D_{\mathrm{pl}} /\left(M c_{\mathrm{p}}\right)$. The third term on the right side of the equation is the Joule - Thomson effect, which can be ignored in the reinjection water pipeline, and the Sukhov temperature drop formula is obtained ${ }^{[29]}$.

By the simultaneous Eqs. (28) to (30) and Eq. (7), Eq. (28), a growth and change model for solid particles in longdistance reinjection water pipelines can be established.

Table 1 Related parameters in the El-Dessouky brine density mode

\begin{tabular}{|c|c|c|c|c|c|}
\hline No. & Parameter & No. & Parameter & No. & Parameter \\
\hline 1 & $F_{2}=A$ & 5 & $A_{1}=4.0322 G_{1}+0.1153 G_{2}+3.26 \times 10^{-4} G_{3}$ & 9 & $G_{1}=0.5$ \\
\hline 2 & $F_{3}=2 A_{2}-1$ & 6 & $A_{2}=-0.1082 G_{1}+1.571 G_{2}-4.23 \times 10^{-4} G_{3}$ & 10 & $G_{2}=B$ \\
\hline 3 & $F_{4}=4 A_{3}-3 A$ & 7 & $A_{3}=-0.01224 G_{1}+1.74 G_{2}-9.0 \times 10^{-6} G_{3}$ & 11 & $G_{3}=2 B-1$ \\
\hline 4 & $A=(2 T-200) / 160$ & 8 & $A_{4}=6.92 \times 10^{-4} G_{1}-8.7 \times 10^{-5} G_{2}-5.3 \times 10^{-5} G_{3}$ & 12 & $B=(2 s a l-150) / 150$ \\
\hline
\end{tabular}

Note: $T$ represents temperature, ${ }^{\circ} \mathrm{C}$. sal represents salinity. 


\section{MODEL SOLUTION}

The key to solving the growth and change model for solid particles in long-distance water-injection pipelines is the solution to the particle population balance equation. To this end, the Direct Quadrature Method of Moments (DQMOM) proposed by Fan [18] can be used for solving.

The moments of the particle distribution function $n(v$, $x, t)$ are approximated by an $n$-point Gaussian integral, as shown in Eq. (34).

$$
m_{k}=\int v^{k} n(v, x, t) \mathrm{d} v \approx \sum_{i=1}^{N} v_{i}^{k} w_{i}
$$

where, $v_{i}$ is abscissas, i.e., the particle scale of the $i$-th point; $w_{i}$ is the weights, indicating the number of particles whose abscissas is $v_{i} ; N$ is the number of integral nodes. $K=0 \sim$ $2 N-1$.

In this case, the particle number density function can also be approximated as Eq. (35).

$$
n(v, x, t) \approx \sum_{i=1}^{N} w_{i} \delta\left(v-v_{i}\right)
$$

where, $\delta$ is the Dirac delta function [30].

By substituting Eq. (35) into Eq. (7), Eq. (36) can be obtained.

$$
\begin{aligned}
& \int\left\{\frac{\partial w_{i} \delta\left(v-v_{i}\right)}{\partial t}+\nabla \cdot\left(V w_{i} \delta\left(v-v_{i}\right)\right)-\nabla \cdot\left(D \nabla\left(w_{i} \delta\left(v-v_{i}\right)\right)\right)\right\} v^{k} \mathrm{~d} v= \\
& =\int S(t, v, x) v^{k} \mathrm{~d} v
\end{aligned}
$$

After Eq. (36) is derived and organized, Eq. (37) can be obtained.

$$
\begin{aligned}
& \int\left\{\delta\left(v-v_{i}\right)\left\{\frac{\partial w_{i}}{\partial t}+\nabla \cdot\left(V w_{i}\right)-\nabla \cdot\left(D \nabla\left(w_{i}\right)\right)\right\}-\right. \\
& -\delta^{\prime}\left(v-v_{i}\right)\left\{w_{i} \frac{\partial v_{i}}{\partial t}+w_{i} V \cdot \nabla v_{i}-\left(D \nabla\left(w_{i}\right) \cdot \nabla v_{i}+\nabla \cdot\left(D w_{i} \nabla v_{i}\right)\right)\right\}- \\
& -\delta^{\prime \prime}\left(v-v_{i}\right)\left\{D w_{i}\left(\nabla v_{i}\right)^{2}\right\} v^{k} \mathrm{~d} v=\int S(t, v, x) v^{k} \mathrm{~d} v
\end{aligned}
$$

Let $\zeta_{i}=v_{i} w_{i}$, and by substituting Eq. (37), we can get Eq. (38).

$$
\begin{aligned}
& \int\left\{\delta\left(v-v_{i}\right)\left\{\frac{\partial w_{i}}{\partial t}+\nabla \cdot\left(V w_{i}\right)-\nabla \cdot\left(D \nabla\left(w_{i}\right)\right)\right\}-\right. \\
& -\delta^{\prime}\left(v-v_{i}\right)\left\{\frac{\partial \zeta_{i}}{\partial t}+\nabla \cdot\left(V \zeta_{i}\right)-v_{i}\left(\frac{\partial w_{i}}{\partial t}+\nabla \cdot\left(V w_{i}\right)-\nabla \cdot\left(D w_{i}\right)\right)\right\}- \\
& -\delta^{\prime \prime}\left(v-v_{i}\right)\left\{D w_{i}\left(\nabla v_{i}\right)^{2}\right\} v^{k} \mathrm{~d} v=\int S(t, v, x) v^{k} \mathrm{~d} v
\end{aligned}
$$

$$
\begin{aligned}
& \left\{\frac{\partial w_{i}}{\partial t}+\nabla \cdot\left(V(v, x) w_{i}\right)-\nabla \cdot\left(D \nabla w_{i}\right)=\right. \\
& =\xi_{i}^{(1)} \frac{\partial \zeta_{i}}{\partial t}+\nabla \cdot\left(V(v, x) \zeta_{i}\right)-\nabla \cdot\left(D \nabla \zeta_{i}\right)= \\
& =\xi_{i}^{(2)} D w_{i}\left(\nabla v_{i}\right)^{2}=\xi_{i}^{(3)}
\end{aligned}
$$

By substituting Eq. (39) into Eq. (38), Eq. (40) can be obtained.

$\int\left\{\sum_{i=1}^{N} \delta\left(v-v_{i}\right) \xi_{i}^{(1)}-\sum_{i=1}^{N} \delta^{\prime}\left(v-v_{i}\right)\left(\xi_{i}^{(2)}-\xi_{i}^{(1)}\right)-\sum_{i=1}^{N} \delta^{\prime \prime}\left(v-v_{i}\right) \xi_{i}^{(3)}\right\} v^{k} \mathrm{~d} v=$

$=\int S(v, x) v^{k} \mathrm{~d} v$

According to the $\delta$ function property [30], Eq. (40) can be transformed into Eq. (41).

$(1-k) \sum_{i=1}^{N} v_{i}^{k} \xi_{i}^{(1)}+k \sum_{i=1}^{N} v_{i}^{k} \xi_{i}^{(2)}+(1-k) k \sum_{i=1}^{N} v_{i}^{k-2} \xi_{i}^{(3)}=\int S(v, x) v^{k} \mathrm{~d} v$

For the solution of $S_{i}^{N}$, the Product-Difference algorithm (PDA) [32] can be used.

Write Eq. (41) as a matrix, see Eq. (42).

$A \alpha=d$

where, $\alpha, A, d$, see Eq. (43), Eq. (44) and Eq. (45) respectively [30].

$\boldsymbol{\alpha}=\left[\xi_{1}^{(1)} \cdots \xi_{N}^{(1)} \xi_{1}^{(2)} \cdots \xi_{N}^{(2)}\right]^{\mathrm{T}}=\left[\begin{array}{c}\xi_{i}^{(1)} \\ \xi_{i}^{(2)}\end{array}\right]$

$A=\left[A_{1}, A_{2}\right]$

$d=A_{3}+\left[\underline{S}_{0}^{N} \cdots \underline{S}_{2 k-1}^{N}\right]$

$A_{1}$ and $A_{2}$ in Eq. (44) and $A_{3}$ in Eq. (45) can be calculated by Eqs. (46) to Eq. (48) respectively.

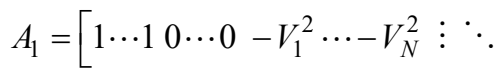

$$
\begin{aligned}
& \left.\vdots 2(1-N) V_{1}^{2 N-1} \cdots 2(1-N) V_{N}^{2 N-1}\right] \\
& A_{2}=\left[\begin{array}{lllllll}
0 & \cdots & 0 & 1 \cdots 1 & 2 V_{1} & \cdots & 2 V_{N}
\end{array} \vdots \cdot \ddots\right. \\
& \left.\vdots 2(N-1) V_{1}^{2 N-2} \cdots 2(N-1) V_{N}^{2 N-2}\right]
\end{aligned}
$$

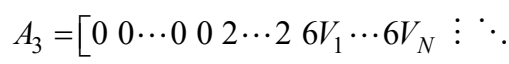

$$
\begin{aligned}
& \left.\vdots 2(N-1)(2 N-1) V_{1}^{2 N-3} \cdots 2(N-1)(2 N-1) V_{N}^{2 N-3}\right]
\end{aligned}
$$

The matrix $\boldsymbol{\alpha}$, i.e. $\xi_{i}^{(1)}$ and $\xi_{i}^{(2),}$ can be obtained by Eq. (49). $\xi_{i}^{(3)}$ can be directly obtained through the starting point and end point particle size data.

$\boldsymbol{\alpha}=A^{-1} d$ 
After $\xi_{i}^{(1)}$ and $\xi_{i}^{(2)}$ are obtained, they can be brought into Eq. (38), and then solved by the Runge Kutta function $\left(\right.$ ode $\left.^{45}\right)$ in Matlab to obtain each moment during the particle motion process [30].

The moment method can only track the overall information on the solid particles along the path, such as the coalescence amount and the average particle size, but cannot reflect the specific distribution of particle size. To this end, Pope [33] proposed a moment-based particle distribution model, see Eq. (50).

$$
n(v, x, t)=\exp \left(\sum_{i=0}^{N-1} A_{i} v^{i}\right)
$$

Perform a moment transformation on Eq. (50) to obtain Eq. (51)

$m_{k}=\int v^{k} \exp \left(\sum_{i=0}^{N-1} A_{i} v^{i}\right) d v \quad k=0,1, \ldots, N-1$

After $m_{k}$ is obtained by the moment method, it is substituted into Eq. (51), $A$ is obtained using NewtonRaphson method, and then substituted into Eq. (50) to figure out the size distribution.

\section{RESULTS AND DISCUSSION \\ 4.1 Model Verification}

(a) Calculating example. The water injection process is adopted in a certain block of Shengli Oilfield, and the schematic diagram of the water-injection pipeline is shown in Fig. 1. The figure shows the ground transportation course of the injected water from the center united station (CUS), through water-injection station (WIS) and the water distributing station (WDS), and finally to the injection well (IW).

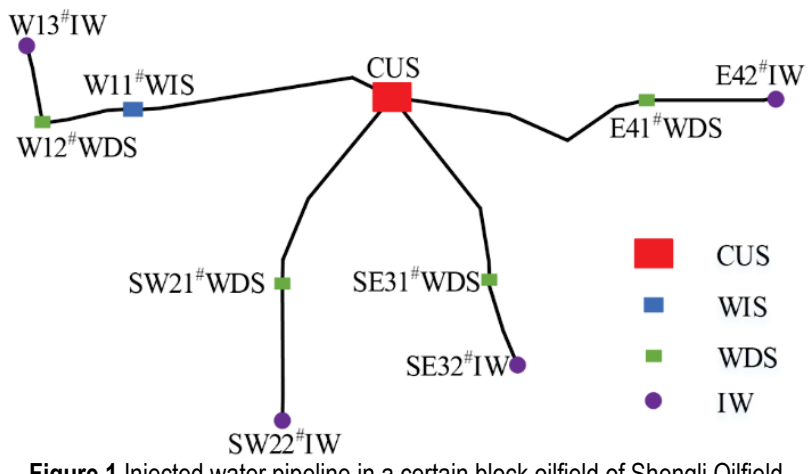

Figure 1 Injected water pipeline in a certain block oilfield of Shengli Oilfield

Table 2 Structural parameters and operating parameters of the water-injection

\begin{tabular}{|c|c|c|c|}
\hline Pipeline Section & $L_{\mathrm{pl}} / \mathrm{m}$ & $D_{\mathrm{pl}} / \mathrm{m}$ & $Q / \mathrm{m}^{3} / \mathrm{d}$ \\
\hline CUS to W11 WIS & 5100 & 0.15 & 1800 \\
\hline W11 $1^{\#}$ WIS to W12 $2^{\#}$ DS & 900 & 0.10 & 460 \\
\hline W12 WDS to W13" IW & 300 & 0.05 & 50 \\
\hline CUS to SW21" WDS & 1250 & 0.10 & 550 \\
\hline SW21" WDS to SW22"IW & 370 & 0.05 & 60 \\
\hline CUS to SE31 $1^{\#}$ WDS & 1600 & 0.10 & 600 \\
\hline SE31 ${ }^{\#}$ WDS to SE32 $2^{\#} \mathrm{~W}$ & 350 & 0.05 & 55 \\
\hline CUS to E41 ${ }^{\#} \mathrm{WDS}$ & 1760 & 0.10 & 610 \\
\hline E41 ${ }^{\#}$ WDS to E42 ${ }^{\#}$ IW & 460 & 0.05 & 50 \\
\hline
\end{tabular}

The pipe length $\left(L_{\mathrm{pl}}\right)$, pipe diameter $\left(D_{\mathrm{pl}}\right)$ and throughout $(Q)$ of the water-injection pipeline involved in Fig. 1 are shown in Tab. 2.

The temperatures of CUS outlet, E41 ${ }^{\#}$ WDS inlet, E42 ${ }^{\#} \mathrm{IW}$ wellhead and the soil at the depth of the pipeline were detected, and the detection results were $43{ }^{\circ} \mathrm{C}, 36{ }^{\circ} \mathrm{C}$, $21{ }^{\circ} \mathrm{C}$, and $11^{\circ} \mathrm{C}$, respectively, as shown in Fig. 2 .

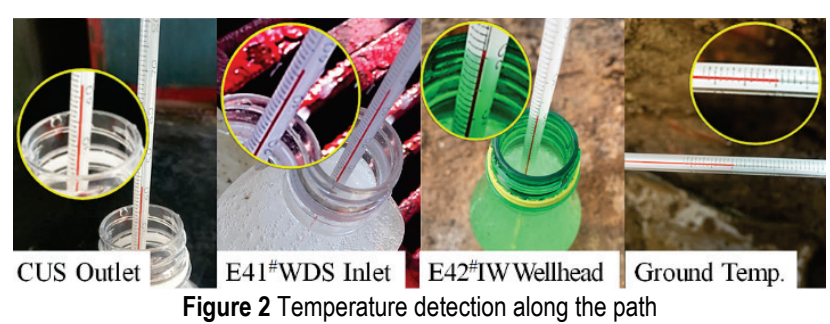

In addition, the total salinity of the injected water at the united station outlet was $0.6 \mathrm{ppt}$. According to the ElDessouky brine density model and the temperature detection result, the injected water density of the united station outlet and the wellhead was $991.1 \mathrm{~kg} / \mathrm{m}^{3}$ and 1000.9 $\mathrm{kg} / \mathrm{m}^{3}$ respectively. The density of injected water of the wellhead change rate was $0.9 \%$ relative to the united station outlet. If the density effect is neglected, the injected water flow in the block oilfield can be regarded as an incompressible and stable flow.

(b) Particle Size Distribution Model. When the Pope model is used, it is a must to meet the premise that the particle size distribution (PSD) conforms to the normal distribution. Gaussian function, Lorentz function and Weber function are commonly used PSD fitting functions, and the fitting results of the detection positions are shown in Fig. 3.
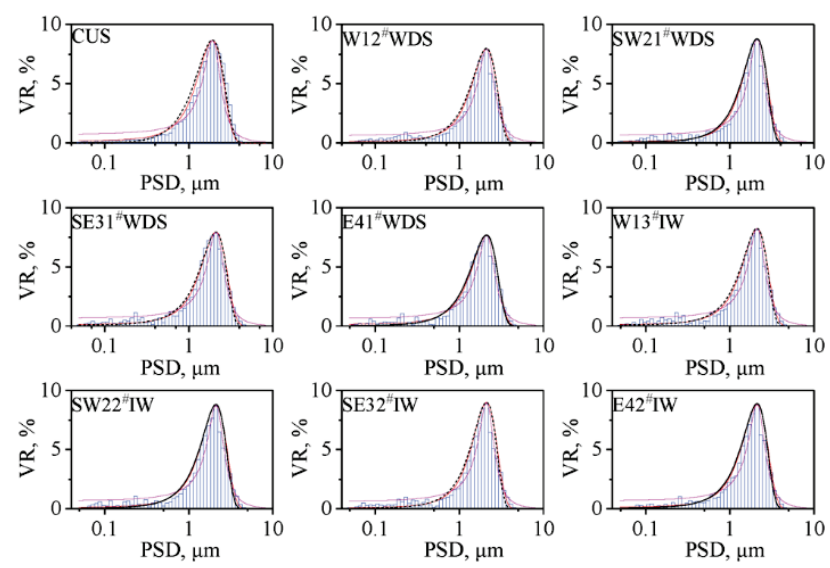

PSD of Solid Particle - Gauss Peak Fit
Lorentz Peak Fit $\quad$ Weber Peak Fit

Figure 3 PSD fitting results at different checkpoints

The calculation results of the fitting degree are shown in Tab. 3.

As can be seen from Tab. 3, the Gaussian model has the highest fitting degree, with the average fitting degree of $97.15 \%$, and the average fitting degree of the Lorentz model and the Weber model is $93.52 \%$ and $96.78 \%$, respectively. Therefore, the particle size distribution conforms to the Gaussian distribution model and satisfies the using conditions of the Pope model. 
Table 3 Fitting degree of size distribution of suspended solid particles / $\%$

\begin{tabular}{|c|c|c|c|c|c|c|c|c|c|c|}
\hline \multirow[b]{2}{*}{ Function } & \multicolumn{9}{|c|}{ Detection position } & \multirow[b]{2}{*}{ Mean } \\
\hline & CUS & $\begin{array}{l}\text { W12 } \\
\text { WDS }\end{array}$ & SW21 ${ }^{\#}$ WDS & $\begin{array}{l}\text { SE31 } \\
\text { WDS }\end{array}$ & $\begin{array}{l}\text { E41\# } \\
\text { WDS }\end{array}$ & $\begin{array}{l}\mathrm{W}^{\prime} 3^{\#} \\
\text { IW }\end{array}$ & $\begin{array}{l}\mathrm{SW}^{\text {SW }}{ }^{\#} \\
\text { IW }\end{array}$ & $\begin{array}{l}\text { SE32 }^{\#} \\
\text { IW }\end{array}$ & $\begin{array}{c}\text { E42 } \\
\text { IW }\end{array}$ & \\
\hline Gauss & 96.92 & 97.67 & 97.81 & 96.46 & 96.26 & 96.96 & 97.63 & 96.91 & 97.79 & 97.15 \\
\hline Lorentz & 91.32 & 94.24 & 92.55 & 93.10 & 92.72 & 94.80 & 94.62 & 94.66 & 93.72 & 93.52 \\
\hline Weber & 96.89 & 96.83 & 97.35 & 97.24 & 96.17 & 96.18 & 96.95 & 96.14 & 97.27 & 96.78 \\
\hline
\end{tabular}

(c) Model Validation. In the liquid-solid two-phase flow model of the water-injection pipeline, the coefficients $C_{1}, C_{2}$ and $n$ in equation (19) and equation (24) could be calculated by linear regression with experimental results. Based on the median diameter detection results of "CUSW12"WDS-W13"IW" and "CUS-E41"WDS-E42"IW", the minimum difference between the calculated median diameter and detected median diameter was regarded as the optimal target, the flow distance of injected water for 2 minutes was the iterative step size, and $C_{1}, C_{2}$, and $n$ were calculated using the particle population method, with the results of $0.43,0.57$, and 1.42 , respectively. Relative to the detection value, the median diameter of $\mathrm{W} 12^{\#} \mathrm{WDS}$, $\mathrm{W} 13^{\#} \mathrm{IW}, \mathrm{E} 41^{\#} \mathrm{WDS}$ and $\mathrm{E} 42^{\#} \mathrm{IW}$ was $4.77 \%, 5.48 \%$, $3.41 \%$, and $5.85 \%$, respectively, and thus, the volume ratio (VR) and the cumulative volume ratio (CVR) of the particle size distribution (PSD) in remaining water distributing stations and the wellheads were calculated, with the results shown in Fig. 4.
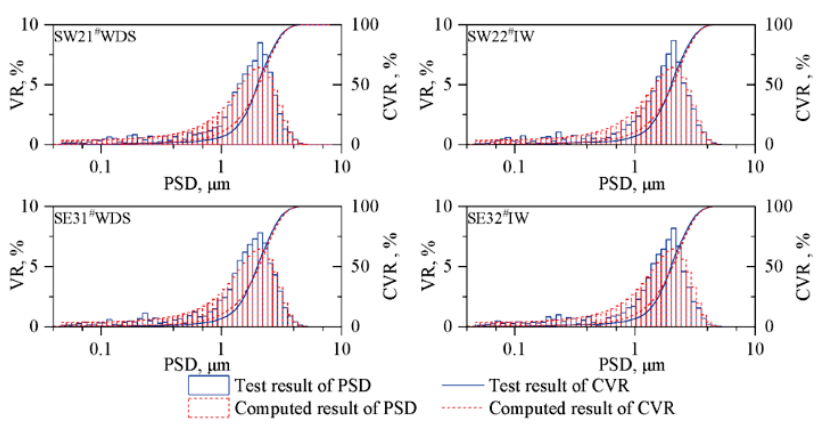

Figure 4 PSD in water distributing stations and wellheads

The calculation results of median diameters of SW21"WDS, SW22" IW, SE31 ${ }^{\#}$ WDS, SE32 ${ }^{\#}$ IW are shown in Tab. 4.

Table 4 Median diameters of water distributing stations and injection wells

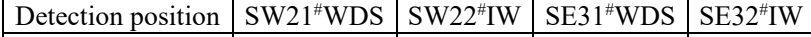

\begin{tabular}{|c|c|c|c|c|}
\hline Detection value & 2.111 & 2.185 & 2.123 & 2.193 \\
\hline Calculated value & 2.018 & 2.097 & 2.035 & 2.110 \\
\hline Relative error / \% & 4.405 & 4.027 & 4.145 & 3.785 \\
\hline
\end{tabular}

As can be seen from Fig. 4 and Tab. 4, compared with the detection values, the liquid-solid two-phase flow model of the water-injection pipeline can accurately calculate PSD of the injected water. Where, the fitting degree ${ }^{[34]}$ of PSD of SW2 $1^{\#}$ WDS, SW22 $2^{\#} \mathrm{IW}$, SE31 3 WDS and SE32 $2^{\#}$ IW were $94.6 \%, 92.2 \%, 92.4 \%$, and $93.4 \%$, respectively, and the average fitting degree error was $6.9 \%$. The relative error of the median diameter was $4.4 \%, 4.0 \%, 4.1 \%$, and $3.7 \%$ respectively, with an average relative error of $4.1 \%$. Therefore, the model of solid particle growth and change in the water-injection pipeline built in this paper can be used to predict the growth and change to solid particles in oilfield injected water.

\subsection{Effect of the CUS Outlet Flow Rate on PSD of the Wellheads}

By taking the water-injected pipeline between the united station and the injection wells as the research object, according to the growth and change model for solid particles in the long-distance water-injected pipeline, the injected water operating conditions (Tab. 2) and the PSD detection result at the united station outlet (Fig. 3), the impact of the flow rate of CUS outlet on the PSD of the wellheads was analyzed, and the calculation results are shown in Fig. 5.

As can be seen from Fig. 5, increased flow rate can suppress the coalescence growth of solid particles in the injected water. The increase in the injected water flow rate can reduce the effective collision probability between the particles, thereby decreasing the growth rate of the particles. This phenomenon is consistent with the experimental phenomenon found by Ho [35]. Ho considered that the collision efficiency $\left(n_{\mathrm{p}}\right)$ between particles is associated with the relative velocity of the particles $\left(\left|v_{\mathrm{p} 1}-v_{\mathrm{p} 2}\right|\right)$, the particle density $\left(\rho_{\mathrm{p}}\right)$, the particle size (small particle $d_{\mathrm{p}}$, large particle $D_{\mathrm{k}}$ ), and the viscosity of the fluid $(\mu)$, and based on this, proposed the corresponding particle collision efficiency model

$n_{\mathrm{p}}=\left(\frac{\Psi_{i}}{\Psi_{i}+0.65}\right)^{3.7}$, where $\Psi_{i}=\rho_{\mathrm{p}}\left|v_{\mathrm{p} 1}-v_{\mathrm{p} 2}\right| d_{\mathrm{p}}^{2} / 18 \mu D_{\mathrm{k}}$.

Therefore, when other conditions are constant, merely increasing the fluid velocity may enlarge the relative velocity between the particles, and reduce the effective collision between the particles, thereby slowing the growth rate of the particles.
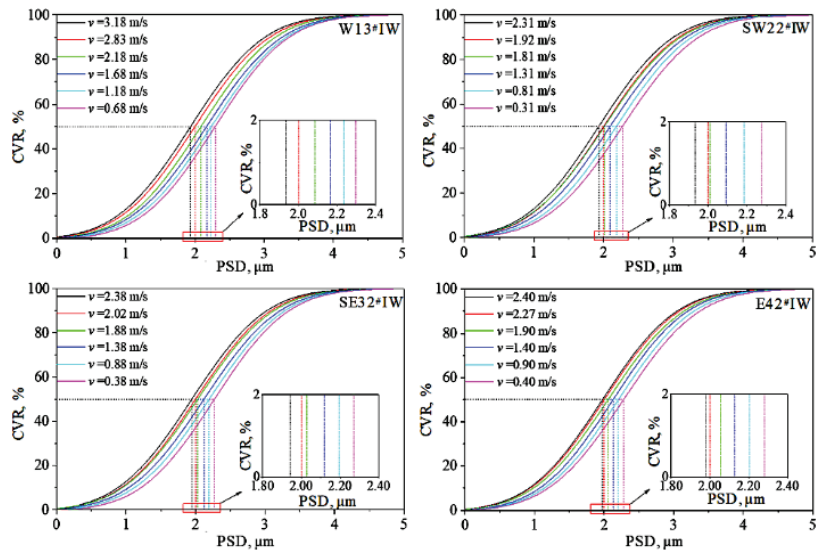

Figure 5 Influence of CUS outlet flow rate on PSD of wellheads

The control index of the median diameter of solid particles in the injected water is less than $2.0 \mu \mathrm{m}$ in the certain block of Shengli Oilfield. According to the temperature and the median diameter of injected water at the outlet of CUS, and the structural parameters of the pipeline (Tab. 2), this model was used to calculate the 
critical outlet flow rate of CUS. When the other conditions remain unchanged, for the injection wells W13 $\mathrm{IW}$, SW22 $2^{\#} \mathrm{IW}, \mathrm{SE} 32^{\#} \mathrm{IW}$ and E42 $\mathrm{IW}$, the critical outbound flow rate of CUS is $2.83 \mathrm{~m} / \mathrm{s}, 1.92 \mathrm{~m} / \mathrm{s}, 2.02 \mathrm{~m} / \mathrm{s}$ and 2.27 $\mathrm{m} / \mathrm{s}$ respectively. Therefore, when the critical outbound flow rate is $2.83 \mathrm{~m} / \mathrm{s}$, the median diameter of the solid particles at the wellheads can satisfy the injection requirement.

\subsection{Effect of the CUS Outlet Temperature on PSD of the Wellheads}

According to the growth and change model for solid particles in long-distance water-injection pipelines, the operating conditions of Tab. 2 and the CUS outlet PSD in Fig. 3, the PSD of the wellheads at different CUS outlet temperatures is calculated. The results are shown in Fig. 6.

As can be seen from Fig. 6, the elevated temperature is conducive to suppressing an increase in the solid particle size. The increase in temperature poses an impact on particle growth and change mainly in two aspects. First, according to Ho [35], although the temperature rise can increase the probability of collision between particles, the effective collision probability between particles decreases instead due to the increased kinetic energy of particles. Second, according to Arrhenius theory [36], the temperature rise can reduce the nucleation rate of solid particles, that is, reduce the particle number. Therefore, under the combined effect of the above reasons, the median diameter of the solid particles gradually decreases with the rise of temperature.
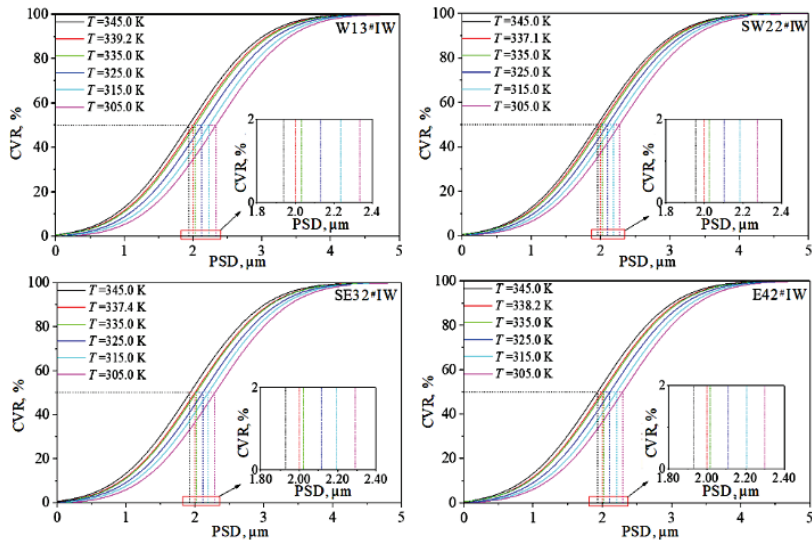

Figure 6 Influence of CUS outlet temperature on PSD of wellheads

Based on the flow rate and the median diameter of injected water at the outlet of CUS, and the pipe parameters, the model was used to calculate the critical outlet temperature of CUS. Through calculation, for the injection wells W13" IW, SW22"IW, SE32" IW, E42" IW, the critical outlet temperature of CUS is $339.2 \mathrm{~K}, 337.1 \mathrm{~K}, 337.4 \mathrm{~K}$, $338.2 \mathrm{~K}$, respectively, that is, the median diameter of the wellhead can be made to satisfy the injection requirement when the CUS outlet temperature reaches $339.2 \mathrm{~K}$.

\subsection{Effect of the CUS Outlet PSD on PSD of the Wellheads}

Due to the different water quality of the produced water per day, the PSD of CUS outlet will also fluctuate. According to the growth and change model for solid particles in the long-distance water-injection pipeline and the injected water operating conditions, the impact of different PSD of CUS outlet on the PSD of the wellheads is calculated, with the results shown in Fig. 7.

In Fig. 7, the particle diameter of the wellheads takes on a rising trend as the particle diameter of the CUS outlet increases. Relative to the impact of the flow rate and temperature of the CUS outlet, the impact of the PSD of CUS outlet on the PSD of the wellheads is more intuitive. When the other conditions remain unchanged, the increase in the median diameter of the solid particles of the outlet, that is, the growth in the number and size of the solid particles in the injected water, increases the collision probability between the particles, resulting in the increase in the median diameter of the solid particles at the wellheads.

According to the flow rate and the temperature of injected water at the outlet of CUS, and the pipe parameters, the model was used to calculate the critical median diameter of CUS outlet. After calculation, for the injection wells W13"IW, SW22" IW, SE32 IW and E42"IW, when the critical median diameter is $1.504 \mu \mathrm{m}, 1.659 \mu \mathrm{m}, 1.599 \mu \mathrm{m}$ and $1.592 \mu \mathrm{m}$ respectively, that is, when the CUS outlet median diameter is $1.534 \mu \mathrm{m}$, the median diameter of the wellheads can meet the injection requirement.
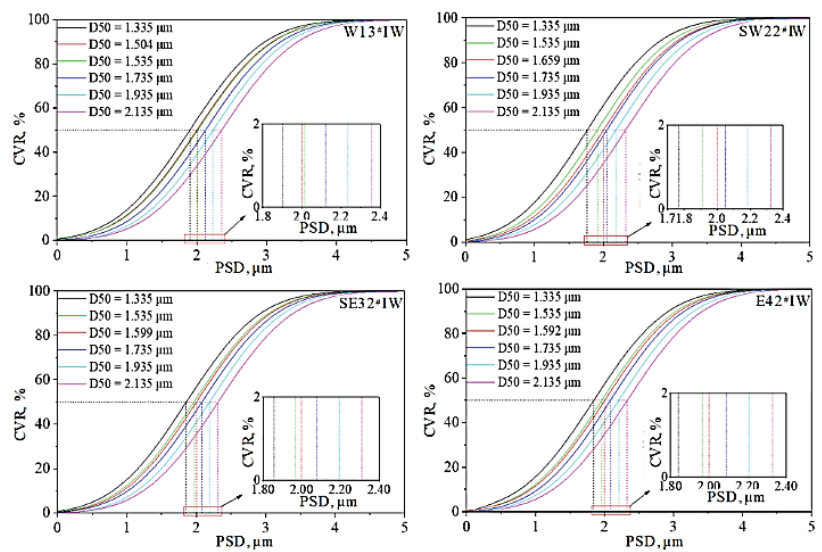

Figure 7 Impact of the PSD of CUS outlet on the PSD of wellheads

In summary, increasing the flow rate, temperature, and median diameter of the united station outlet can reduce the solid particle diameter at the wellheads. When the critical flow rate of the CUS outlet is $2.83 \mathrm{~m} / \mathrm{s}$, or the temperature is $339.2 \mathrm{~K}$, or the median particle size is $1.504 \mu \mathrm{m}$, the median diameter at the wellheads can meet the injection requirement.

\section{CONCLUSION}

To study the growth and change to solid particles in long-distance water-injection pipeline, based on onedimensional fluid control equation and particle population balance equation, a model for the growth and change to solid particles in long-distance water-injection pipeline was established in this paper. This model treats the particles as the continuous media accompanied with water flow and the growth, coalescence, fragmentation and deposition of particles are considered. Comparison of the field test results indicated that the average error of the fitting degree of PSD calculated by the model is $6.9 \%$, and the average relative error of median diameter is $4.1 \%$. Based on water-injection operation in a certain block of Shengli Oilfield, the model was used to predict the critical flow rate, temperature and 
particle diameter of the united station outlet, respectively $2.83 \mathrm{~m} / \mathrm{s}, 339.2 \mathrm{~K}$ and $1.504 \mu \mathrm{m}$, so that the median diameter of the solid particles at the wellhead of the injection well meets the injection requirement. The establishment of this model cannot only be used for the study on the growth and change to solid particles in waterinjection pipelines flowing along water, but also provide a technical reference for the study on the growth and change to low-concentration solid particles in long-distance liquid/gas pipelines.

However, the model was constructed from the perspective of the long-distance water-injection pipeline, ignoring the impact of special pipe section, such as the vertical/inclined pipe section going into and out of the stations and the header section of the water distributing station. Further in-depth study will be needed if the impact of these pipe sections on the growth and change to solid particles or the deposition of solid particles there is taken into account.

\section{REFERENCES}

[1] Igunnu, E. T. \& Chen, G. Z. (2014). Produced water treatment technologies. International Journal of Low-Carbon Technologies, 9(3), 157-177. https://doi.org/10.1093/ijlct/cts049

[2] Kumar, S., Naiya, T. K., \& Kumar, T. (2018). Developments in oilfield scale handling towards green technology: A review. Journal of Petroleum Science and Engineering, 169, 428-444. https://doi.org/10.1016/j.petrol.2018.05.068

[3] Feng, Q., Chen, H., Wang, X. et al. (2016). Well control optimization considering formation damage caused bysuspended particles in injected water. Journal of Natural Gas Science and Engineering, 35, 21-32. https://doi.org/10.1016/j.jngse.2016.08.040

[4] Ghafori, H. \& Ebrahimi, H. R. (2018). Numerical and experimental study of an innovative pipeline design in a granular pneumatic-conveying system. Particuology, 38(2018), 196-203. https://doi.org/10.1016/j.partic.2017.07.007

[5] Song, G., Li, Y., Wang, W. et al. (2018). Numerical simulation of pipeline hydrate particle agglomeration based on population balance theory. Journal of Natural Gas Science and Engineering, 51(2018), 251-261. https://doi.org/10.1016/j.jngse.2018.01.009

[6] Baldyga, J. \& Orciuch, W. (2001). Barium sulphate precipitation in a pipe - an experimental study and CFD modelling. Chemical Engineering Science, 56(7), 2435-2444. https://doi.org/10.1016/S0009-2509(00)00449-8

[7] Tchen, C. (1947). Mean value and correlation problems connected with the motion of small particles suspended in a turbulent fluid. Netherlands: Delft University.

[8] Crowe, C., Sharma, M., \& Stock, D. (1977). The ParticleSource-In Cell (PSI-Cell) Model for Gas-Droplet Flows. Journal of Fluids Engineering, 99(2), 325. https://doi.org/10.1115/1.3448756

[9] Soo, S. (2018). Particulates and Continuum-Multiphase Fluid Dynamics: Multiphase Fluid Dynamics. New York: CRC Press. https://doi.org/10.1201/9780203744291

[10] Spalding, D. B. (1972). Mathematical Models of Continuous Combustion. New York: Springer. https://doi.org/10.1007/978-1-4684-1998-6_1

[11] Bowen, R. (1980). Incompressible porous media models by use of the theory of mixtures. International Journal of Engineering Science, 18(9), 1129-1148. https://doi.org/10.1016/0020-7225(80)90114-7

[12] Pack, D. J., Parks, D. W., \& Chesnoy, A. B. (2012). Gas pipeline preferential site selection occurrence for elemental sulphur \& other particle matter formation \& deposition. Journal of Petroleum Science and Engineering, 94-95(9), 1218. https://doi.org/10.1016/j.petrol.2012.06.022

[13] Leporini, M., Terenzi, A., Marchetti, B. et al. (2019). Experiences in numerical simulation of wax deposition in oil and multiphase pipelines Theory versus reality. Journal of Petroleum Science and Engineering, 174(3), 997-1008. https://doi.org/10.1016/.jpetrol.2018.11.087

[14] Hulburt, H. \& Katz, S. (1964). Some problems in particle technology: A statistical mechanical formulation. Chemical Engineering Science, 19(8), 555-574. https://doi.org/10.1016/0009-2509(64)85047-8

[15] Mungall, J. \& Su, S. (2005). Interfacial tension between magmatic sulfide and silicate liquids Constraints on kinetics of sulfide liquation and sulfide migration through silicate rocks. Earth and Planetary Science Letters, 234(1), 135-149. https://doi.org/10.1016/j.epsl.2005.02.035

[16] Abegg, C. F., Stevens, J. D., \& Larson, M. A. (1968). Crystal size distributions in continuous crystallizers when growth rate is size dependent. AIChE Journal, 14(1), 118-122. https://doi.org/10.1002/aic.690140121

[17] Wang, K., Yu, S., \& Peng, W. (2019). A novel moment method using the log skew normal distribution for particle coagulation. Journal of Aerosol Science, 134(2019), 95-108. https://doi.org/10.1016/j.jaerosci.2019.04.013

[18] Fan, R., Marchisio, D., \& Fox, R. (2004). Application of the direct quadrature method of moments to polydisperse gassolid fluidized beds. Powder Technology, 139(1), 7-20. https://doi.org/10.1016/j.powtec.2003.10.005

[19] Park, S. \& Lee, K. (2002). Analytical solution to change in size distribution of polydisperse particles in closed chamber due to diffusion and sedimentation. Atmospheric Environment, 36(35), 5459-5467. https://doi.org/10.1016/S1352-2310(02)00673-8

[20] Bourdillon, A., Verdin, P., \& Thompson, C. (2016). Numerical simulations of drop size evolution in a horizontal pipeline. International Journal of Multiphase Flow, 78(2016), 44-58. https://doi.org/10.1016/j.jmultiphaseflow.2015.09.009

[21] Michael, F. \& Harris, S. (1987). Aerosol dynamics modeling using the method of moments. Journal of Colloid and Interface Science, 118(1), 252-261. https://doi.org/10.1016/0021-9797(87)90454-1

[22] Pratsinis, S. (1988). Simultaneous nucleation, condensation, and coagulation in aerosol reactors. Journal of Colloid and Interface Science, 124(2), 416-427. https://doi.org/10.1016/0021-9797(88)90180-4

[23] Park, S., Lee, K., Otto, E. et al. (1999). The log-normal size distribution theory of brownian aerosol coagulation for the entire particle size range: Part I : Analytical solution using the harmonic mean coagulation kernel. Journal of Aerosol Science, 30(1), 3-16. https://doi.org/10.1016/S0021-8502(98)00037-8

[24] Randolph, A. Theory of Particulate Processes: Analysis and Techniques of Continuous Crystallization. Pittsburgh, Academic Press.

[25] Park, S., Lee, K., Otto, E. et al. (1997). The log-normal size distribution theory of Brownian agglomeration for the entire particle size range. Journal of Aerosol Science, 28(S1). https://doi.org/10.1016/S0021-8502(97)85013-6

[26] Su, Junwei, Gu, et al. (2007). Solution of population balance equation using quadrature method of moments with an adjustable factor. Chemical Engineering Science, 62(21), 5897-5911. https://doi.org/10.1016/j.ces.2007.06.016

[27] Ma, L., Duan, F., Song, G. et al. (2018). A concentration measurement model of suspended solids in oilfield reinjection water based on underwater scattering. Measurement, 117(2018), 125-132. https://doi.org/10.1016/j.measurement.2017.11.061

[28] El-Dessouky, H. \& Ettouney, H. (2002). Fundamentals of Salt 
Water Desalination. Amsterdam, Elsevier.

[29] Cai, H., Zhao, L., Chen, X. et al. (2018). Optimization of Temperature Drop Formula for Annular Gathering Pipeline in High Water Cut Oil Region of Central Hebei Province. Journal of Power and Energy Engineering, 06(9), 21-26. https://doi.org/10.4236/jpee.2018.69003

[30] Thein, F. (2012). On the Efficiency and Condition of the Core Routine of the Quadrature Methods of Moments(QMOM). Magdeburg, Otto-von-Guericke-Universität Magdeburg.

[31] Junwei, S., Lin, G. U. Z., \& Yun, L. (2006). Study on Direct Quadrature Method of Moment for Population Balance Equation in Isotropic Particle System. Chemical Reaction Engineering and Technology, 22(4), 310-316. https://doi.org/10.1080/02786829708965471

[32] McGraw, R. (1997). Description of Aerosol Dynamics by the Quadrature Method of Moments. Aerosol Science and Technology, 27(2), 255-265.

[33] Pope, S. (1980). Probability distributions of scalars in turbulent shear flow. Symposium on Turbulent Shear Flows, 7-16.

[34] Kasuya, E. (2019). On the use of $r$ and r-squared in correlation and regression. Ecological Research, 34(1), 235-236. https://doi.org/10.1111/1440-1703.1011

[35] Ho, C. A. \& Sommerfeld, M. (2002). Modelling of microparticle agglomeration in turbulent flows. Chemical Engineering Science, 57(15), 3073-3084. https://doi.org/10.1016/S0009-2509(02)00172-0

[36] Lewis, A., Seckler, M., Kramer, H. et al. (2015). Industrial Crystallization: Fundamentals and Applications. UK, Cambridge University Press. https://doi.org/10.1017/CBO9781107280427

\section{Contact information:}

\section{Guiliang LI}

(Corresponding author)

School of Petroleum Engineering, Southwest Petroleum University, CNPC Key Laboratory of Oil \& Gas Storage and Transportation,

Southwest Petroleum University,

Chengdu 610500, China

E-mail: liguiliang_spu@163.com

\section{Changjun LI}

School of Petroleum Engineering, Southwest Petroleum University, CNPC Key Laboratory of Oil \& Gas Storage and Transportation,

Southwest Petroleum University,

Chengdu 610500, China

\section{Jie WANG}

School of Petroleum Engineering, Southwest Petroleum University CNPC Key Laboratory of Oil \& Gas Storage and Transportation,

Southwest Petroleum University,

Chengdu 610500, China

\section{Nanning XIA}

PetroChina Kunlun Gas Co., Ltd. Jiangsu Branch,

Nantong 226100, China

\section{Xiuling CHEN}

PetroChina Changqing Oilfield Company Third Gas Production Plant, Ordos 017000, China 